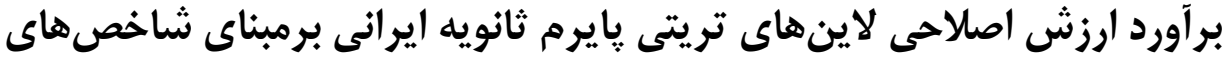

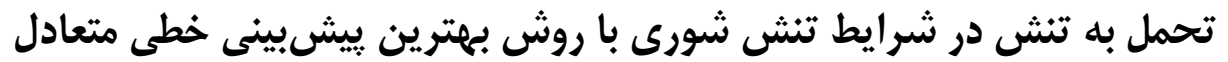

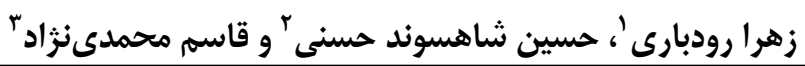

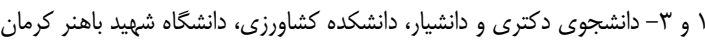

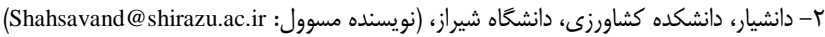

تاريخ دريافت:

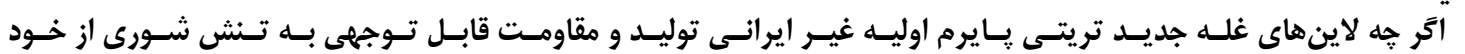

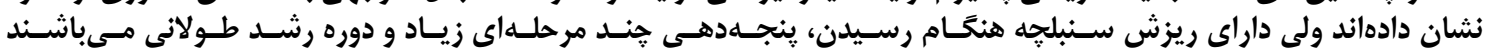

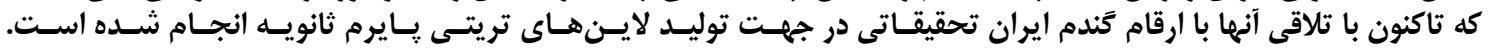

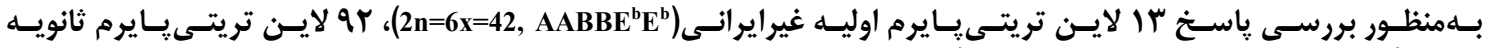
اير انى (1-6)" (1-6)"

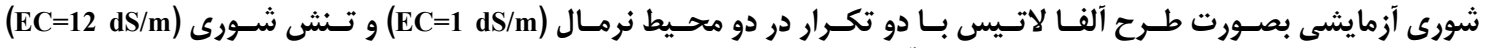

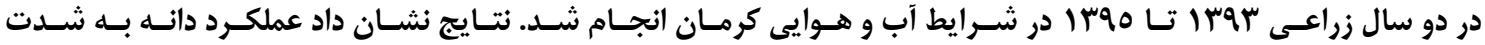

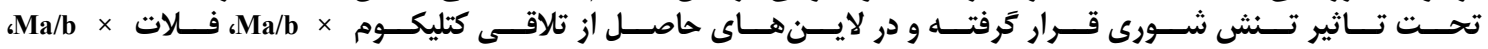

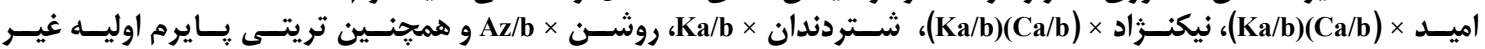

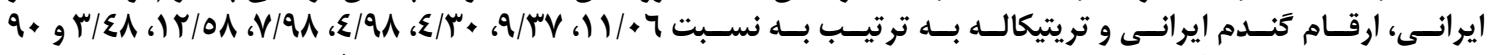

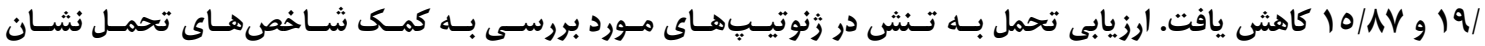

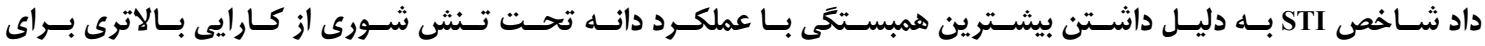

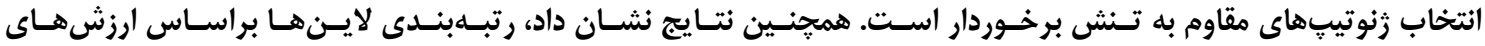

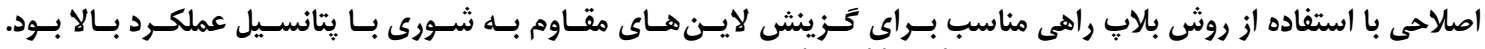

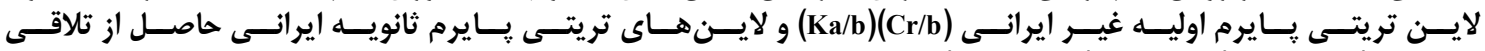

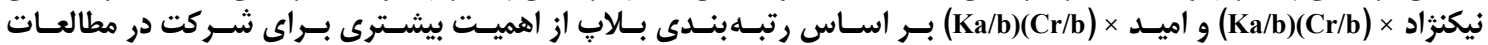

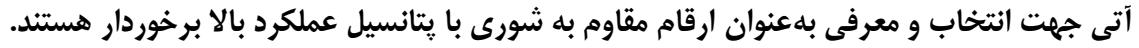

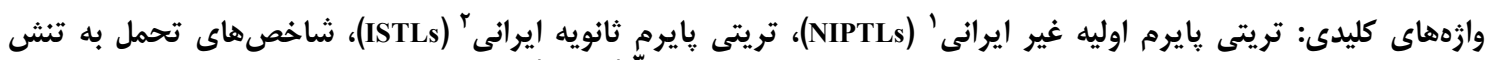

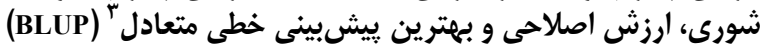

برگها در ارتباط با تحمل به شورى مورد توجه قرار مى گئيرد.

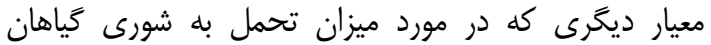

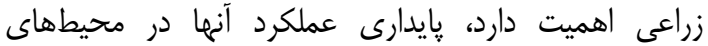

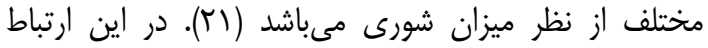

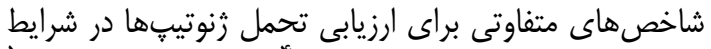

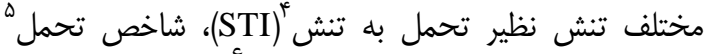

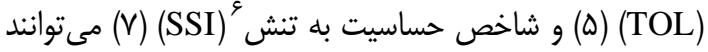

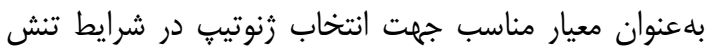

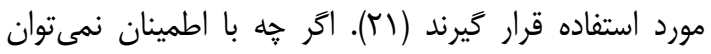

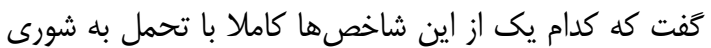

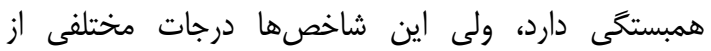

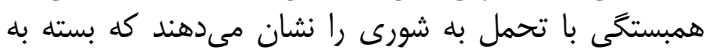

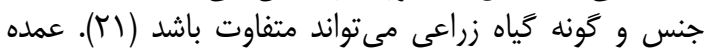

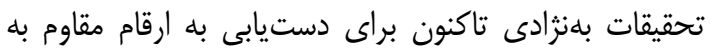

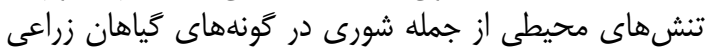

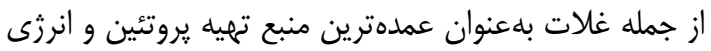

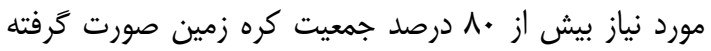

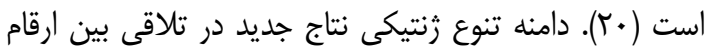

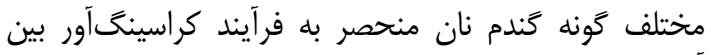

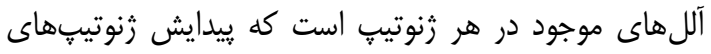

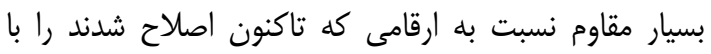

1- Non Iranian primary Tritipyrum Lines

3- Best Linear Unbiased Prediction

5- Tolerance index

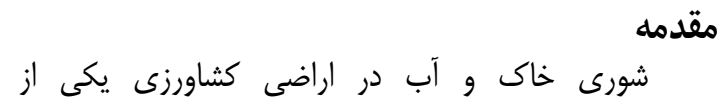

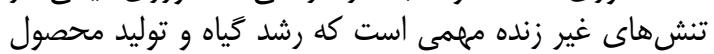

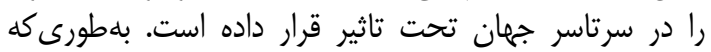

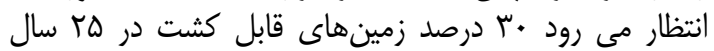

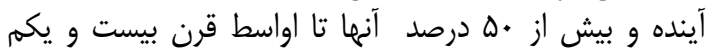

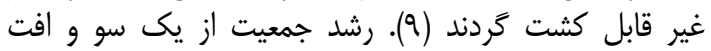

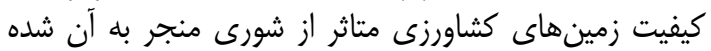

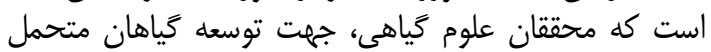

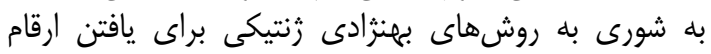

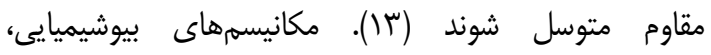

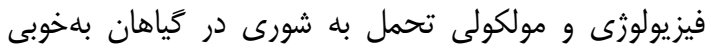

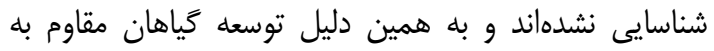

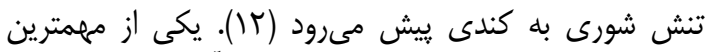

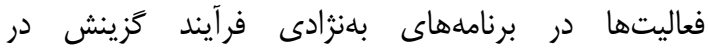

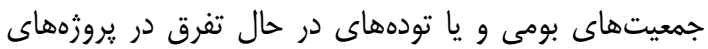

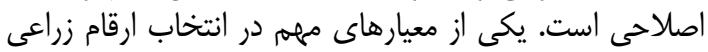

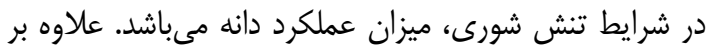

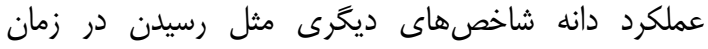

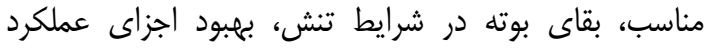
همبسته با عملكرد دانه و يا تجمع يك يون يناص خاص در ساقه و

2- Iranian Secondary Tritipyrum Lines

4- Stress tolerance index

6-Stress susceptibility index 
حاصل از انتخاب با اين روش براى برخى از صفات

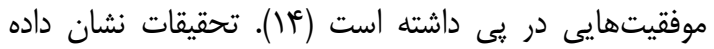

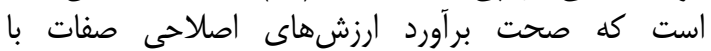
وراثتيذيرى بالا نسبت به صفات با با وراثتيذيرى إيايين بيشتر

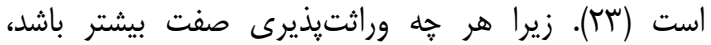

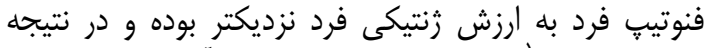

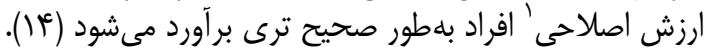

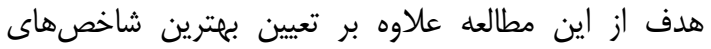

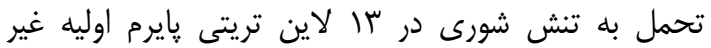

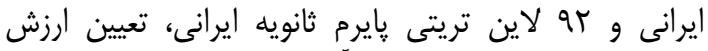

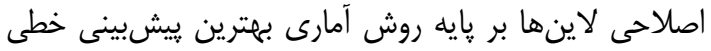

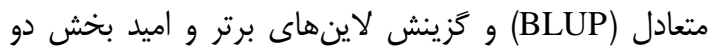
نوع تريتى پايرم مىباشد.

\section{مواد و روشها}

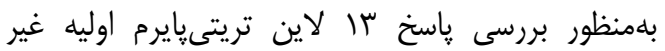

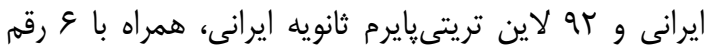

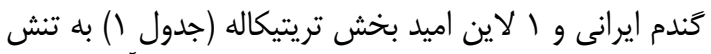

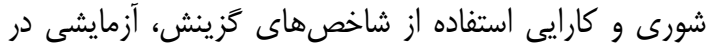

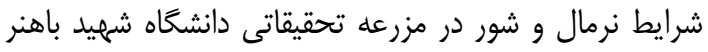
كرمان (ECw=0.8dS/m, ECs=1dS/m) و منطقه اختيار آباد كرمان

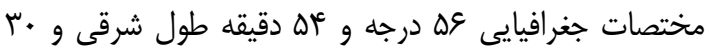

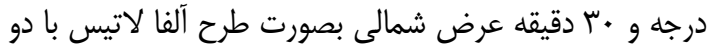

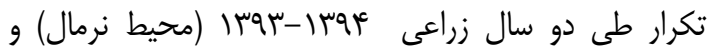

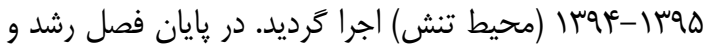

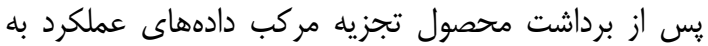

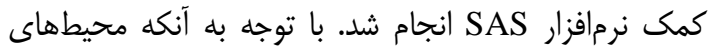

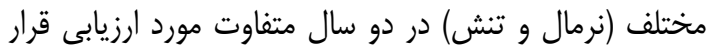

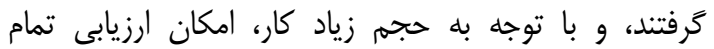

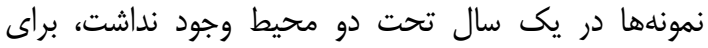

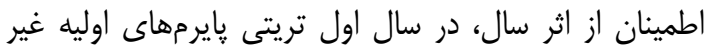

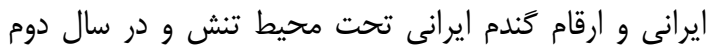

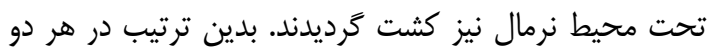

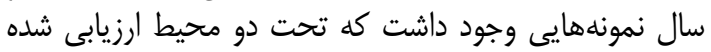

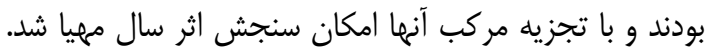

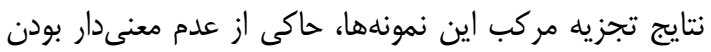

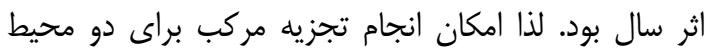

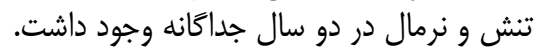

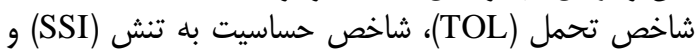
شاخص تحمل به تنش (STI) با روابط زير محاسبه شدند. $\mathrm{TOL}=\mathrm{Y}_{\mathrm{n}}-\mathrm{Y}_{\mathrm{s}}$

$\mathrm{SSI}=\left[1-\left(\mathrm{Y}_{\mathrm{S}} / \mathrm{Y}_{\mathrm{n}}\right)\right] / \mathrm{SI}$

$\mathrm{STI}=\left(\mathrm{Y}_{\mathrm{n}} \times \mathrm{Y}_{\mathrm{s}}\right) /\left(\hat{\mathrm{Y}}_{\mathrm{n}}\right)^{2}$

$\mathrm{SI}=\left[1-\left(\hat{\mathrm{Y}}_{\mathrm{s}} / \hat{Y}_{\mathrm{n}}\right)\right]$

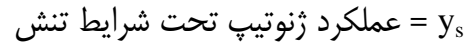

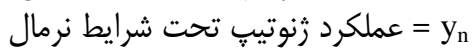

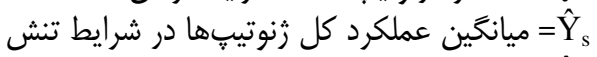

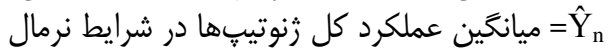

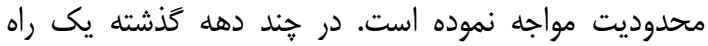

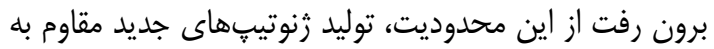

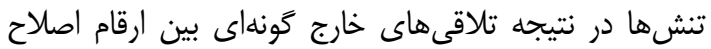

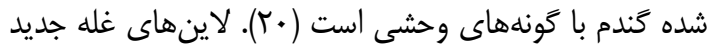

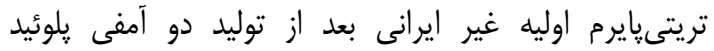

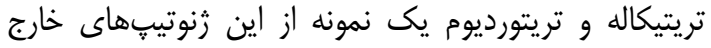

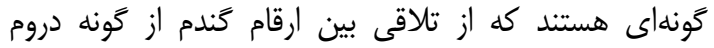

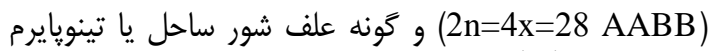
بسارابيكوم (2n=2x=14 E Eb

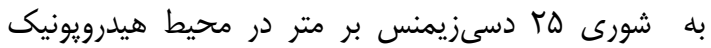

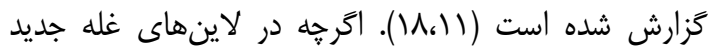

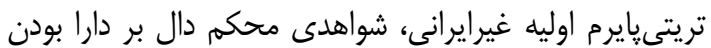

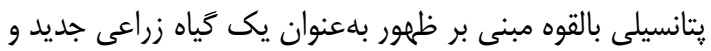

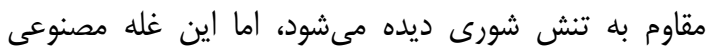

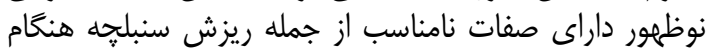

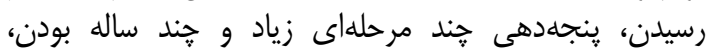

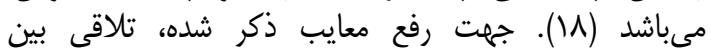

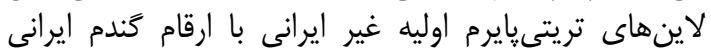

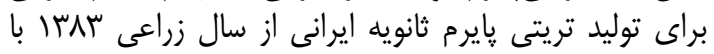

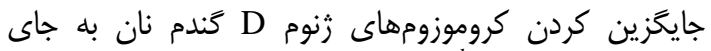

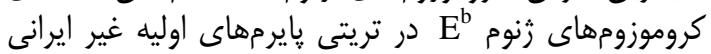
(2n=6x=42, AABBE $\left.{ }^{b} E^{b}\right)$

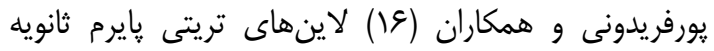

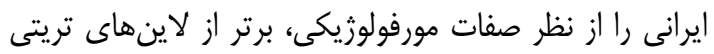

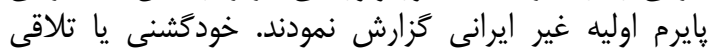

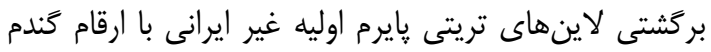

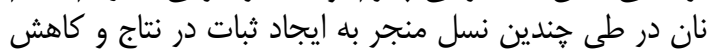

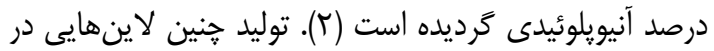

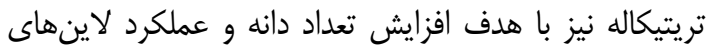

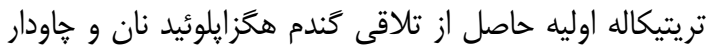

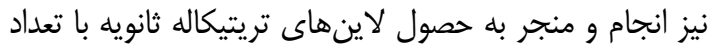

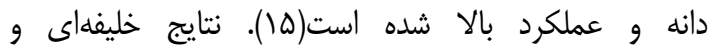

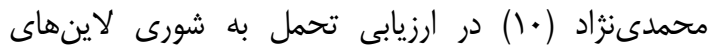

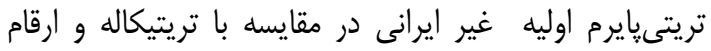

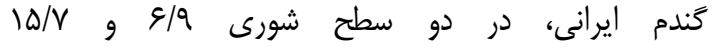

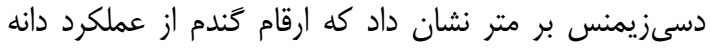

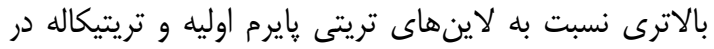

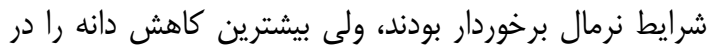

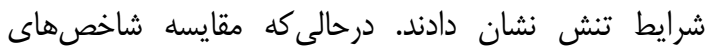

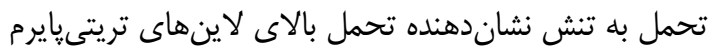

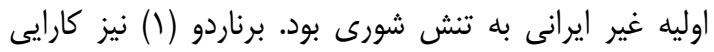

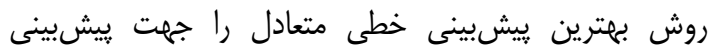

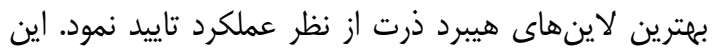

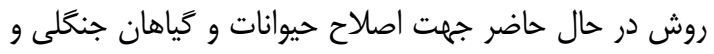

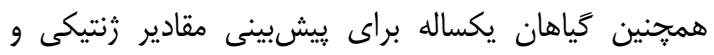

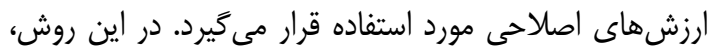

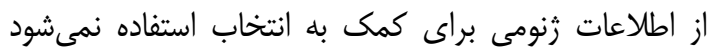

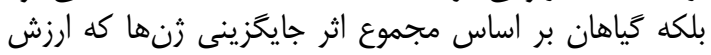

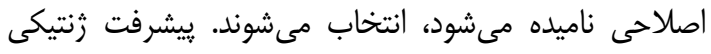


Table 1. Codes of genotypes used in this study

جدول I- كد مربوط به نمونههاى مورد مطالعه

\begin{tabular}{|c|c|c|c|c|c|c|c|c|}
\hline كد & 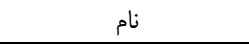 & 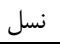 & كد & 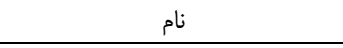 & نسل & 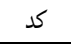 & نام & 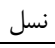 \\
\hline $\mathrm{G} 1$ & 1. Ma/bx كتليكوم (CM) & 9 & G42 & 6. (Ka/b)(Cr/b) اميد) & 9 & G83 & 12.Az/bx روشن & 8 \\
\hline G2 & 2. Ma/b× كتليكوم & 9 & G43 & 7. (Ka/b)(Cr/b) اميد & 9 & G84 & 13.Az/bx روشن & 8 \\
\hline G3 & 3. Ma/b× كتليكوم & 9 & G44 & 8. (Ka/b)(Cr/b) اميد & 9 & G85 & 14.Az/bx روشن & 8 \\
\hline G4 & 4. Ma/b× Maكوم & 9 & G45 & 9. (Ka/b)(Cr/b) اميد & 9 & G86 & 15.Az/bx روشن & 8 \\
\hline G5 & 5. Ma/b× كتليكوم & द & G46 & 10. (Ka/b)(Cr/b) اميد) & 9 & G87 & 16.Az/bx روشن & 8 \\
\hline G6 & 6تليكوم_ Ma/b. كا & v & G47 & 11. (Ka/b)(Cr/b) اميد× & 9 & G88 & 17.Az/bx روشن & 9 \\
\hline G7 & 7. Ma/b× Maكوم & v & G48 & 12. (Ka/b)(Cr/b) اميد) & 8 & G89 & 18.Az/bx روشن & 8 \\
\hline G8 & 8. Ma/b× Maكوم & v & G49 & 13. (Ka/b)(Cr/b) اميد) & 9 & G90 & 19.Az/bx روشن & 8 \\
\hline G9 & 9. Ma/b× كتليكوم & $v$ & G50 & 14. (Ka/b)(Cr/b) اميد× & 9 & G91 & 20. Az/bx روشن & 9 \\
\hline G10 & 10. Ma/bx كتليكوم & v & G51 & 15. (Ka/b)(Cr/b) اميد× & 9 & G92 & 21.Az/bx روشن & 9 \\
\hline G11 & 1. Ma/bx فلات ( FM) & 9 & G52 & 16. (Ka/b)(Cr/b) اميد) & 9 & G93 & $(\mathrm{Ka} / \mathrm{b})(\mathrm{cr} / \mathrm{b})-4$ & r. \\
\hline G12 & 2. Ma/bx Mلات & $\varepsilon$ & G53 & 1. (Ka/b)(Cr/b) × نيك نزاد (NKC) & $\checkmark$ & G94 & $(\mathrm{ma} / \mathrm{b})(\mathrm{cr} / \mathrm{b})-1$ & $r$. \\
\hline G13 & 3. Ma/bx Mلات & \& & G54 & 2. $(\mathrm{Ka} / \mathrm{b})(\mathrm{Cr} / \mathrm{b})$ × نيك نزاد & $v$ & G95 & $(\mathrm{ma} / \mathrm{b})(\mathrm{cr} / \mathrm{b})-2$ & r. \\
\hline G14 & 4. Ma/b× فلات & 9 & G55 & 3. $(\mathrm{Ka} / \mathrm{b})(\mathrm{Cr} / \mathrm{b})$ × نيك نزاد & $\checkmark$ & G96 & $\mathrm{Az} / \mathrm{b}$ & r. \\
\hline G15 & 5. Ma/bx Maل & $\varepsilon$ & G56 & 4. $(\mathrm{Ka} / \mathrm{b})(\mathrm{Cr} / \mathrm{b}) \times$ & $\checkmark$ & G97 & $\mathrm{Ka} / \mathrm{b}$ & $r$. \\
\hline G16 & 6. Ma/b× فلات & 9 & G57 & 5. $\quad(\mathrm{Ka} / \mathrm{b})(\mathrm{Cr} / \mathrm{b}) \times$ نيك نزاد & $v$ & G98 & $\mathrm{La} / \mathrm{b}$ & r. \\
\hline G17 & 7. Ma/bx Mلات & 9 & G58 & 6. $(\mathrm{Ka} / \mathrm{b})(\mathrm{Cr} / \mathrm{b}) \times$ نيك نزاد & v & G99 & $\mathrm{St} / \mathrm{b}$ & $r \cdot$ \\
\hline G18 & 8. Ma/bx فلات & s & G59 & 7. $(\mathrm{Ka} / \mathrm{b})(\mathrm{Cr} / \mathrm{b}) \times$ & v & G100 & $(\mathrm{Ka} / \mathrm{b})(\mathrm{Cr} / \mathrm{b})-1$ & r. \\
\hline G19 & 9. Ma/b× فلات & v & G60 & 8. $\quad(\mathrm{Ka} / \mathrm{b})(\mathrm{Cr} / \mathrm{b}) \times$ نيك نزاد & $v$ & G101 & $(\mathrm{Ka} / \mathrm{b})(\mathrm{Cr} / \mathrm{b})-2$ & $r \cdot$ \\
\hline G20 & 10. Ma/bx فلات & v & G61 & 9. $(\mathrm{Ka} / \mathrm{b})(\mathrm{Cr} / \mathrm{b}) \times$ & v & G102 & $(\mathrm{Ka} / \mathrm{b})(\mathrm{Cr} / \mathrm{b})-3$ & $r \cdot$ \\
\hline G21 & 11. Ma/bxali & v & G62 & 10. $(\mathrm{Ka} / \mathrm{b})(\mathrm{Cr} / \mathrm{b}) \times$ نيك نزاد & v & G103 & $(\mathrm{St} / \mathrm{b})(\mathrm{Cr} / \mathrm{b})$ & r. \\
\hline G22 & 12. Ma/b×الات & v & G63 & 11. $(\mathrm{Ka} / \mathrm{b})(\mathrm{Cr} / \mathrm{b}) \times$ نيك نزاد & $v$ & G104 & $\mathrm{La}(4 \mathrm{~b} 4 \mathrm{~d}) / \mathrm{b}$ & $r$. \\
\hline G23 & 13. Ma/b×اتلات & v & G64 & 12. $(\mathrm{Ka} / \mathrm{b})(\mathrm{Cr} / \mathrm{b}) \times$ نيك نزاد & v & G105 & $\mathrm{Cr} / \mathrm{b}$ & $r$. \\
\hline G24 & 14. Ma/b× فلات & v & G65 & 13. $(\mathrm{Ka} / \mathrm{b})(\mathrm{Cr} / \mathrm{b}) \times$ نيك نزاد & v & G106 & 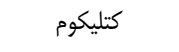 & - \\
\hline G25 & 15. Ma/bx فلات & v & G66 & 1. Ka/b×شتردندان ( SK) & 9 & G107 & فلات & - \\
\hline G26 & 16. Ma/b×اتلات & v & G67 & 2. Ka/b× شتردندان & 9 & G108 & 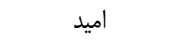 & - \\
\hline G27 & 17. Ma/b×فلات & $\checkmark$ & G68 & 3. Ka/b× شتردندان & 9 & G109 & نيك نزاد & - \\
\hline G28 & 18. Ma/b× فلات & $v$ & G69 & 4. Ka/b× شتردندان & 9 & G110 & 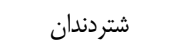 & - \\
\hline G29 & 19 Ma/bxali & v & G70 & 5. Ka/b×شتردندان & 9 & G111 & ل رشن & - \\
\hline G30 & 20 Ma/b×فلات & v & G71 & 6. Ka/bx شتردندان & 9 & G112 & تريتيكاله (M45) & - \\
\hline G31 & فلات & v & G72 & 1. Az/bx روشن (RA) & 9 & & & \\
\hline G32 & 22 Ma/b×فلات & $v$ & G73 & 2. Az/bx روشن & 9 & & & \\
\hline G33 & 23 Ma/bxali & v & G74 & 3. Az/bx روشن & 9 & & & \\
\hline G34 & 24 Ma/b×فلات & v & G75 & 4. Az/bx روشن & 9 & & & \\
\hline G35 & 25 Ma/b×فلات & v & G76 & 5. Az/bx روشن & 9 & & & \\
\hline G36 & فلات 26. Ma/ba & v & G77 & روشن 6. Az/bx & 9 & & & \\
\hline G37 & $\begin{array}{l}\text { 1. (Ka/b)(Cr/b) اميد) } \\
(\mathrm{OKC})\end{array}$ & $\varepsilon$ & G78 & 7. Az/bx روشن & 9 & & & \\
\hline G38 & 2. (Ka/b)(Cr/b) اميد & 9 & G79 & 8. Az/bx روشن & 9 & & & \\
\hline G39 & 3. (Ka/b)(Cr/b) اميد× & 8 & G80 & 9. Aوشن Az/bx & 9 & & & \\
\hline G40 & 4. (Ka/b)(Cr/b) اميد ( & 9 & G81 & 10. Az/bx روشن & 9 & & & \\
\hline G41 & 5. (Ka/b)(Cr/b) اميد) & 9 & G82 & 11. Az/bx روشن & 9 & & & \\
\hline
\end{tabular}




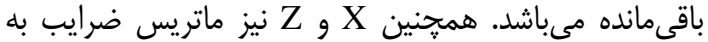
ترتيب براى اثرات ثابت و تصادفى مىباشندا

عملكرد صفتى كمى است كه مكان تانيسم كنترل زنتيكى

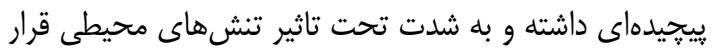

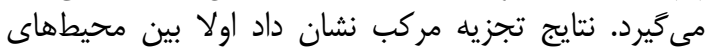

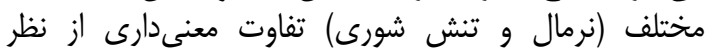

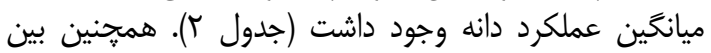

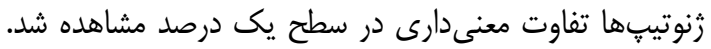

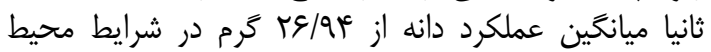

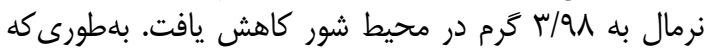

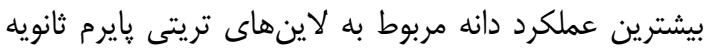

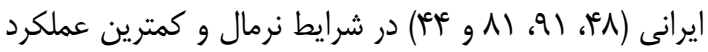

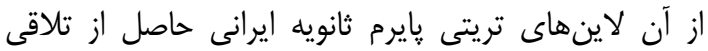

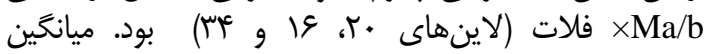

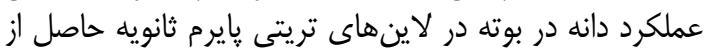

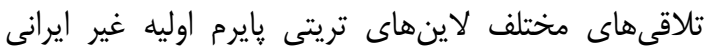

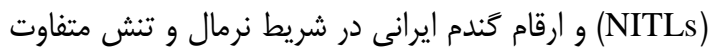

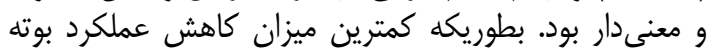

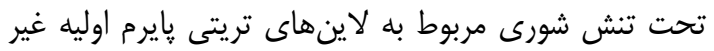

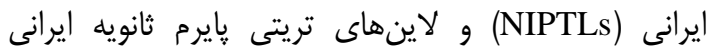

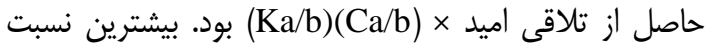

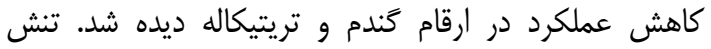

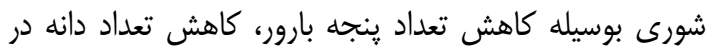

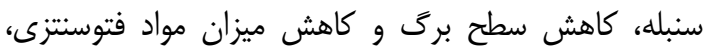

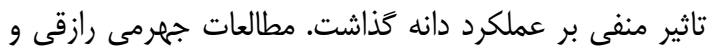

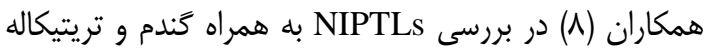

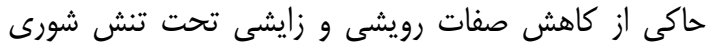

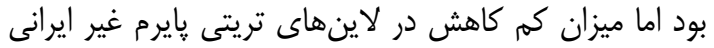

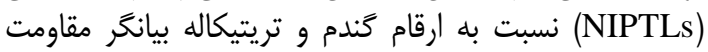
بيشتر آنها نسبت به ارقام و لاين هاى مورد مطالعه بود.

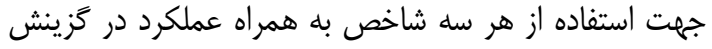

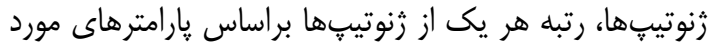

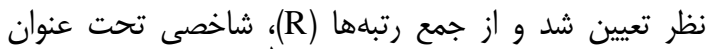

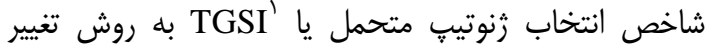

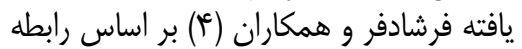

\section{TGSI $=\mathrm{RY}_{\mathrm{s}}+\mathrm{RY}_{\mathrm{n}}+\mathrm{RTOL}+\mathrm{RSSI}+\mathrm{RSTI}$}

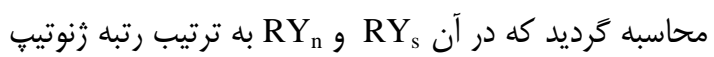
بر اساس عملكرد در محيط تنش و و نرمال است است.

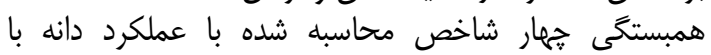

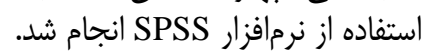

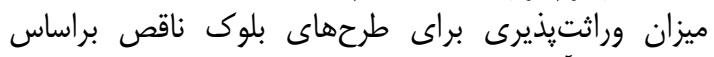
رابطه زير برآورد كرديد:

$h^{2}=\sigma_{g}^{2} /\left(\sigma_{g}^{2}+\sigma^{2} / r\right)$

كه درآن واريانس زنتيكى و فنوتيبى مطابق روابط زير محاسبه

$$
\sigma_{g}^{2}=\frac{m s_{t}-\sigma^{2}}{\left(\frac{k}{k+1}\right) r}
$$

$\sigma_{p}^{2}=\sigma_{g}^{2}+\sigma^{2} / r$

در اين روابط، K تعداد بلوك ناقص و r تعداد تكرار مىباشد.

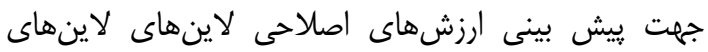

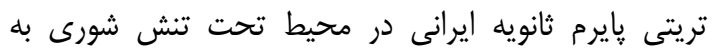

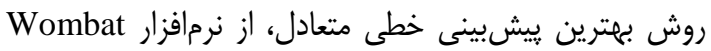

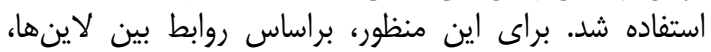

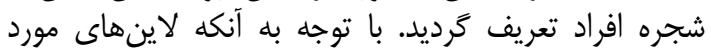

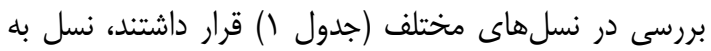

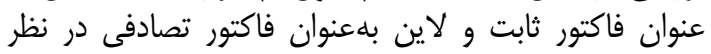
$\mathrm{y}=\mathrm{Xb}+\mathrm{Zu}+\mathrm{e}$

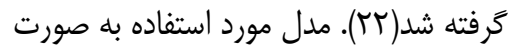

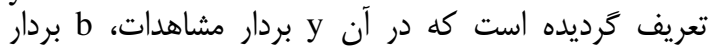
اثرات ثابت، u بردار اثرات تصادفى و e بردار اثرات تصادفى مثرات

Table 2. combined analysis of variance for seed yield of samples used in this study

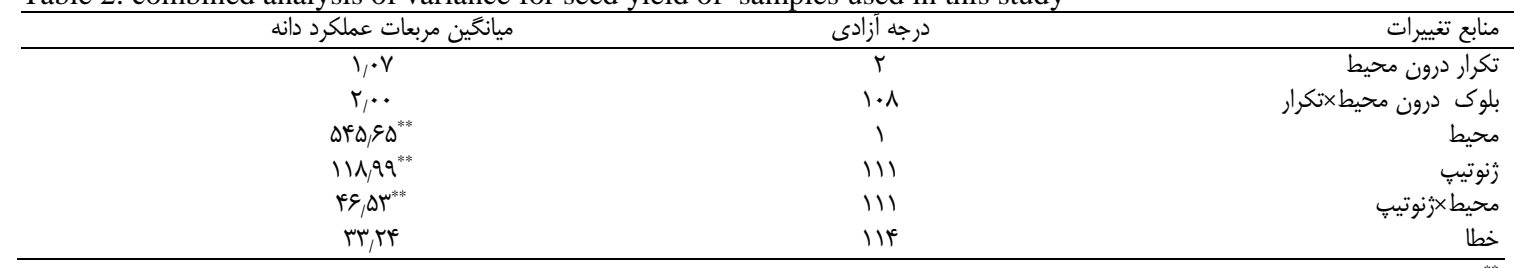

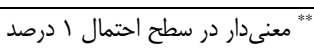

شاخص حساسيت بيشتر از يك، جزء تياهان رساس

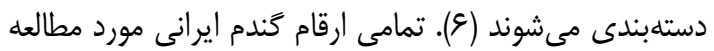

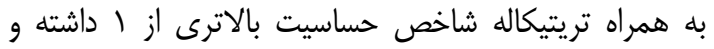

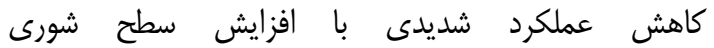
نشان دادند. اين نتايج با نتايج شاهسوند
شاخص هاى تحمل مقادير كم شاخص حساسيت به تنش شمل شورى (SSI) درا

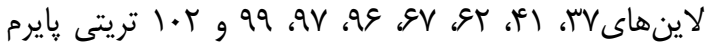
ثانويه ايرانى نشاندهنده حساسيت كمتر اين لاينها

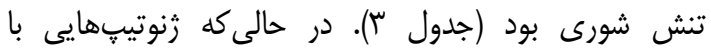




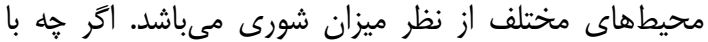

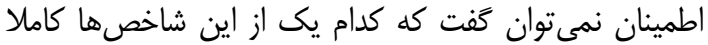

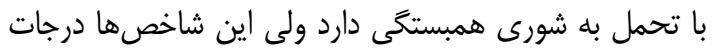

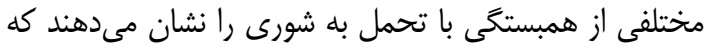

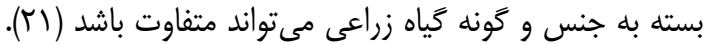

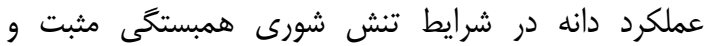

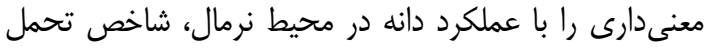

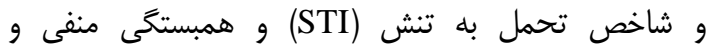
معنى دارى با دو شاخص حساسيت به تهن تنش و شاخص انتخ انتخاب

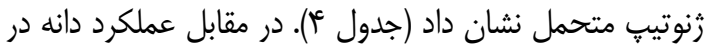

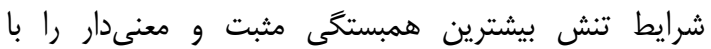

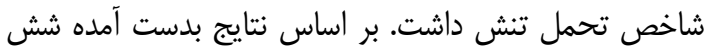

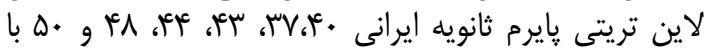

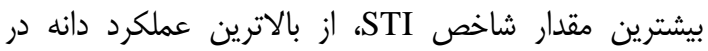

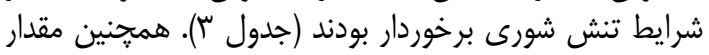

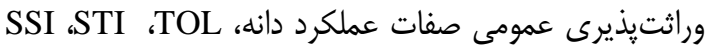

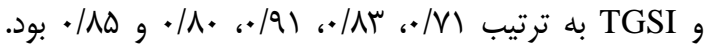

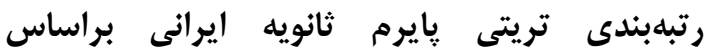
ارزشهاى اصلاحى إنيل

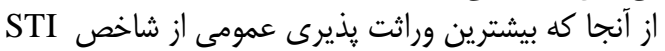

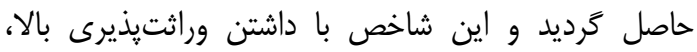

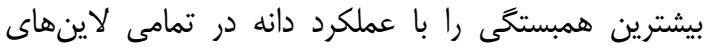

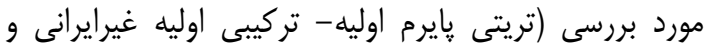

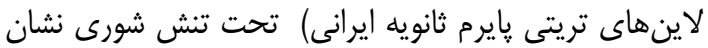

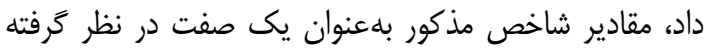

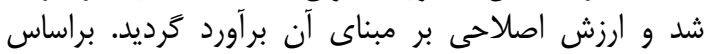

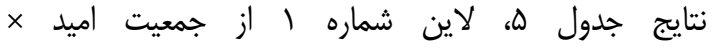

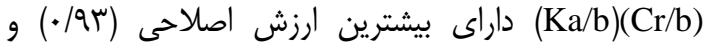

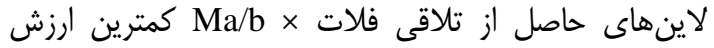

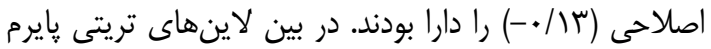

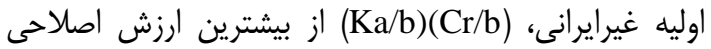

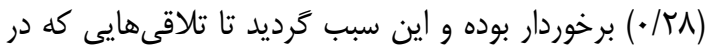

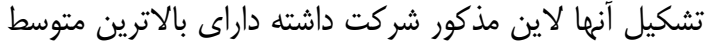

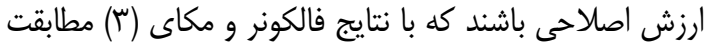

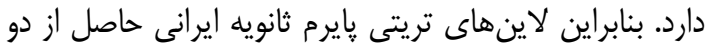

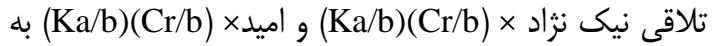

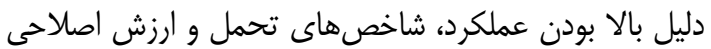

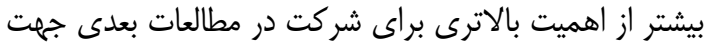

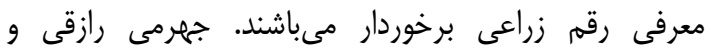

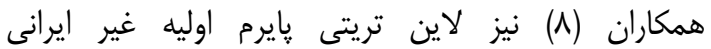

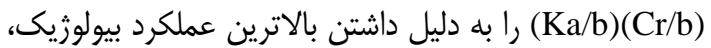

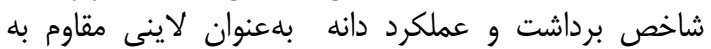

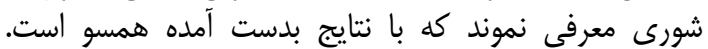

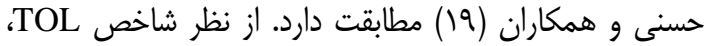

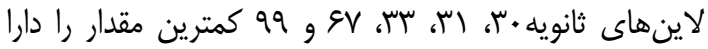

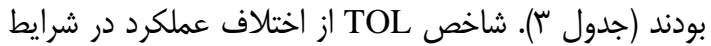

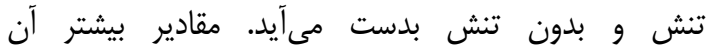

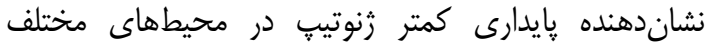

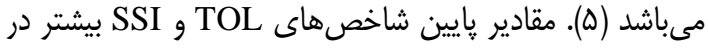

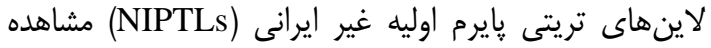

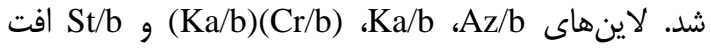

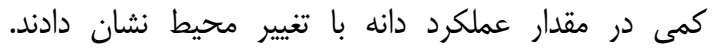

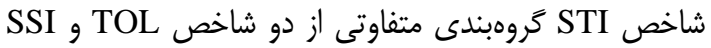

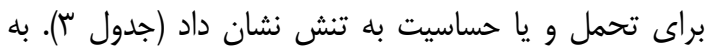

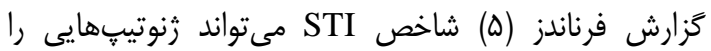

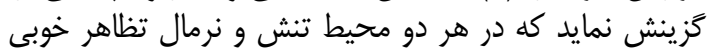

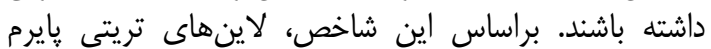

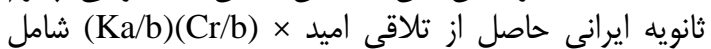

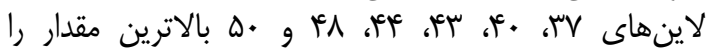

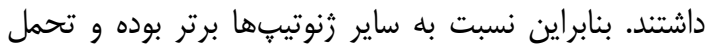

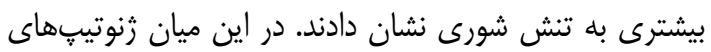

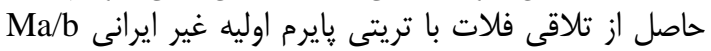

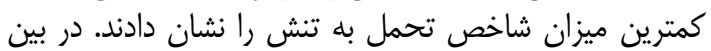

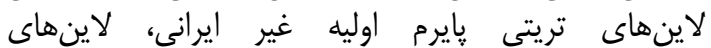

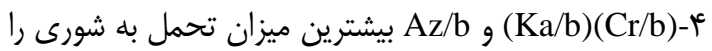

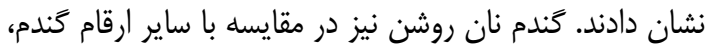

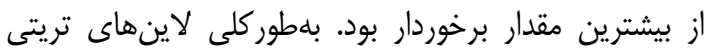

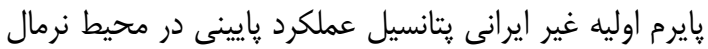

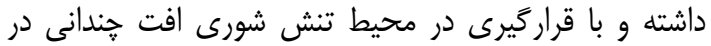

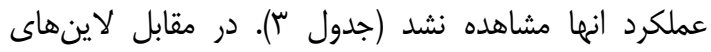

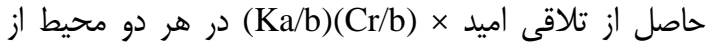

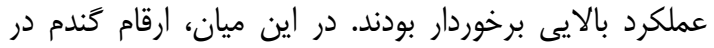

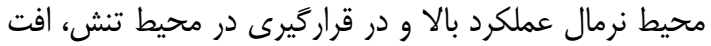

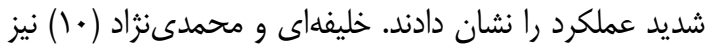

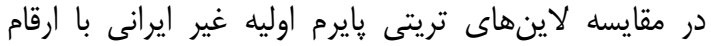

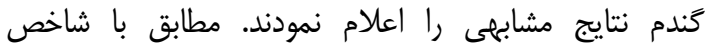
TGSI

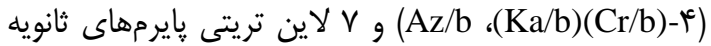

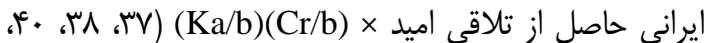

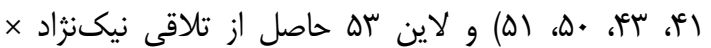

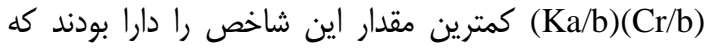

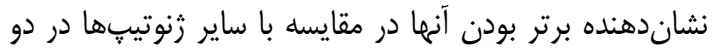

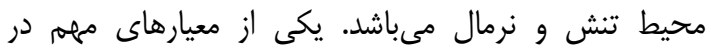

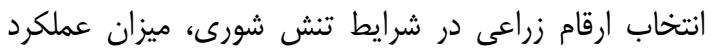

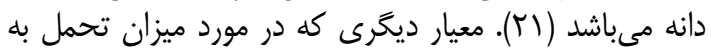

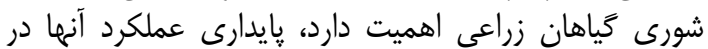


Table 3. Average yield and stress tolerance indices of samples used in this study

جدول ب- متوسط عملكرد و شاخصهاى تحمل به تنش در نمونههاى مورد مطالعه

\begin{tabular}{|c|c|c|c|c|c|c|c|c|c|c|c|c|c|c|c|c|c|c|c|c|c|c|c|c|c|c|c|}
\hline رُنوتيب & Ys & $\mathrm{Yn}$ & TOL & STI & SSI & TGSI & رثنوتيب & Ys & $\mathrm{Yn}$ & TOL & STI & SSI & TGSI & رنوتيّي & Ys & $\mathrm{Yn}$ & TOL & STI & SSI & TGSI & رنوتيب & Ys & $\mathrm{Yn}$ & TOL & STI & SSI & TGSI \\
\hline G1 & $\Gamma / \Delta{ }^{\prime}$ & $r \Delta / \Lambda$. & Tr/TV &.$/ N$ &.$/ 99$ & req & G29 & $1 / \wedge \Delta$ & $|g / N|$ & $14 / 45$ & $\% \bullet$ & $1 / . r$ & $r \mu \mu^{2}$ & G57 & $91 \cdot \Delta$ & $19 / 99$ & $4 . / 91$ & זצא/. & $1 / \cdot \cdot$ & $r \cdot \Lambda$ & G85 & $9 \pi$. & $F \cdot N G$ & $\Gamma Y / \Delta S$ & 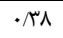 & ./9V & $r .1$ \\
\hline G2 & $r / l$. & Fi/NA & rq/q1 & זו/. & $1 / .9$ & rтq & G30 & V/A৯ & $\Delta / \wedge \Delta$ & יתוז. &.$/ .1$ & . Ne & TथD & G58 & $r / r$. & $1 F /$ & $11 / \Delta$. & $\% \vee v$ &.$/ 9$. & req & G86 & ه"r & TS/QT & $r Y / \Delta V$ & $\cdot \pi r$ & $1 / \cdot 1$ & r"q \\
\hline G3 & $T / F \Delta$ & $r \cdot N \cdot$ & TNTA &.$/ 1$ & 11.9 & $r \cdot r$ & $\mathrm{G} 31$ & $1 / 9$. & $\Delta / \Gamma \varepsilon$ & $r / \Delta \Delta$ &.$/ 1$ & . Na & r.q & G59 & $r / r$. & 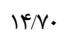 & $\mid r / F$ &.$/ . \Delta$ & . /av & $r .$. & G87 & $1 /$. & TE/IS & צו/ &.$/ 4$ & $1 / 1$. & fir \\
\hline G4 & $r / \cdot \Delta$ & $r \bar{r} / q T$ & rr/Aq &.$/ \cdot 1$ & $1 / \infty$ & MTY & G32 & $1 / 1$. & $\Delta / \wedge \Delta$ & $\varphi / \Delta$ & .1 .1 &.$/ 9$. & res & G60 & $1 / \wedge \Delta$ & $r g / \pi$ & & $\% \vee v$ & $v \cdot v$ & TrV & G88 & $r / . \Delta$ & & $r \mid / . q$ &.$/ . v$ & $1 / .0$ & TrY \\
\hline G5 & $r / F^{e}$ & $r \& / \Delta q$ & $r \in / 1 q$ & $\cdot /$ & $1 / .0$ & $r \cdot r$ & G33 & $1 / \Delta$. & $\Delta / 11$ & r/sD &.$/ 1$ & . $/ \lambda \mid$ & M & G61 & $F_{N}$ & $r g / T$ & TI/DI & .119 & . & r. & G89 & $1 / 1$. & שथ/ & שr/אr &.$/ 4$ & $1 / 1$. & ral \\
\hline G6 & $1 / \Delta \omega$ & צ צואץ & $r \mid / \Lambda)$ & .1 .0 & $1 / \cdot v$ & $r \Delta q$ & G34 &.$/ \%$ & $\Delta / 1$. & $\Delta / M$ & $\%$ & WIF & $r_{\Delta \Delta}$ & G62 & $v / r$ & $\| \pi / \Delta$. & $V / r$. &.$/ 19$ & $\cdot / \Delta V$ & 191 & G90 & $r /$. & $\Delta V / \Delta Y$ & $\Delta F / N T$ & $\cdot / T V$ & $1 / .9$ & $T N G$ \\
\hline G7 & $\cdot N \Delta$ & $r r / \Delta q$ & Tr/AF & r.r & $1 / 1$ & rTS & G35 & $1 / . \cdot$ & $\Delta / \wedge \Delta$ & $\leftarrow / \Delta \Delta$ &.$/ .1$ & سو/. & чs. & G63 & r/A. & $1 F / 1 \mathrm{~V}$ & $\| / T V$ & .1 .9 & . & $r v$. & G91 & D/q. & $\Delta 81.9$ & $\Delta \cdot / 19$ & $\cdot / \Delta$ & $1 / . \mu$ & Mif \\
\hline G8 & $r / T \Delta$ & $r \Delta / 1$. & TY/AD & .1 .9 & $1 / .0$ & זוץ & G36 & $1 /$. & $8 / 9 \Lambda$ & $\Delta / 9 \Lambda$ &.$/ 1$ &.$/ 91$ & $r q v$ & G64 & r/A. & $1 \pi /$. & $N T$. & $\% \bullet \vee$ & . ra & זTr & G92 & $r / \Delta$. & $r \mu / T F$ & TMYF & rr. &.$/ 99$ & grr \\
\hline G9 & $1 /{ }^{\infty} \Delta$ & $r \mid r q q$ & rQ/Ar &.$/ \cdot v$ & $1 / 1$. & rv. & G37 & $r \% 1.0$ & $\Delta N \mathcal{N q}^{2}$ & rT/AF & I/ I & .194 & 90 & G65 & $r / 9 \Delta$ & $1 \% / \pi f$ & $N \Delta Q$ & .1 .9 & . Na & rI. & G93 & $8 / 90$ & TI/DA & س & rאן. &.$N A$ & $19 V$ \\
\hline $\mathrm{G} 10$ & $r / .$. & $r / / \Delta \Delta$ & WNID & $\cdot /$ &.$/ 99$ & Tr & G38 & QND & TNGI & TNNS & $\cdot / \Delta V$ & . & ifs & G66 & $r / q$. & rq/. & rg/A. & זות. & $1 / r^{c}$ & TAF & G94 & r/gD & $r / M V$ & $r \cdot / \Delta r$ & .1 .9 & $1 / . r$ & TAD \\
\hline G11 & $1 / N \Delta$ & ITST & $1 / /{ }^{\circ} \wedge$ &.$/ . r$ & $1 / 4$ & r r & G39 & NYS & rq/19 & $r \cdot /$ r & . $/ \pi V$ & . Ar & 199 & G67 & $r / r$. & $r / \propto q$ & $1 / 19$ &.$/ .1$ & 年 & TAY & G95 & $r / . \cdot$ & INAD & $19 / 90$ & .1 .9 & $1 / \mu$ & riq \\
\hline G12 & $I / N \Delta$ & IVISD & $10 / 9$. &.$/ . \Delta$ & $1 / 4$ & TFe & $\mathrm{G} 40$ & $I T / A \Delta$ & rV/q & $\Gamma \Delta / \cdot \Lambda$ & $\cdot N^{e}$ & .NG & 119 & G68 & $1 / r \Delta$ & $1 . / 95$ & Q/9V &.$/ . r$ & $V \cdot r$ & هצD & G96 & $\mid r / \cdot$ & $r \cdot \pi \cdot$ & $N \pi$. & 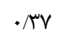 &.$/ F$ & 1.9 \\
\hline $\mathrm{G} 13$ & T/Ta & $15 / 9$. & $\mid r / F D$ & .1 .0 & .91 & $r .9$ & $\mathrm{G} 41$ & $1 . \%$ & $19 / T 1$ & $9 / / \Lambda$ &.$/ r q$ & $\cdot / \Delta \Delta$ & $M \Lambda$ & G69 & 你 & 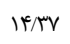 & $\mid r / \cdot r$ & $\% \Delta$ & .99 & ras & G97 & $1 \cdot / \lambda$ & $V|q|$ & $r / / 9$ & . & 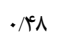 & $19 \pi$ \\
\hline G14 & $1 / N$ & WN. & $\mid \mathrm{V} / \cdot$. & $.1 . \Delta$ & $1 / * \varphi$ & $r \Delta r$ & $\mathrm{G} 42$ & $\mathrm{v} / \mathrm{N}^{\circ}$ & $\Delta \cdot /^{e} V$ & $r \psi / N V$ &.$/ \Delta F$ & .91 & 119 & G70 & $r / s$. & $|r / F|$ & $11 / \cdot 1$ & $\%$ & . & rq. & G98 & r/s. & $T T^{E / g}$. & $r / .$. & זו/. &.$/ 91$ & TeF \\
\hline G15 & $1 / \wedge$ & $1 Q / \pi{ }^{2}$ & $\mid r / \mu c e$ & .1 .4 & $1 / \cdot 1$ & זrו & $\mathrm{G} 43$ & $1 . / \Delta 8$ & $\leftarrow \varepsilon / q$. & reTre & $\cdot N \Delta$ & . /19 & If. & G71 & $1 / \Delta \Delta$ & $r . / .9$ & TNAF & $\% \vee v$ & $1 / .9$ & rgr & G99 & $r \mu$. & $V|q|$ & $F / T$ & .1 .4 & .194 & rar \\
\hline G16 & 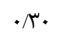 & $I V / M$ & $I V / \Delta \Lambda$ &.$/ .1$ & r// & $r \Delta \Delta$ & G44 & $1 . \%$ & $\Delta \Delta / / \Delta$ & $\Gamma \Delta / / \Delta$ & ./Af & . $/ 9 F$ & $10 \Lambda$ & $\mathrm{G} 72$ & $1 / 9 \Delta$ & reNV & $r \varphi / A r$ &.$/ 15$ & //. & זrז & G100 & r/FD & FT/Tr & $r V / \Delta V$ & $\cdot \pi$ & $1 / \cdot r$ & Try \\
\hline G17 & $\cdot / N \Delta$ & $\mid \phi / 1 \Delta$ & $\| \leftarrow \pi$. &.$/ . r$ & $1 / \cdot 1$ & ila & G45 & V/A. & $\mathrm{eV} / \mathrm{s}$. & ra/s. &.$/ \Delta F$ & .99 & IVe & $\mathrm{G} 73$ & $|/ N|$ & זיזוז & IV/AT & .1 .9 & $1 / 00$ & MeI & G101 & NGD & \$T/ & r/s. & . & $\cdot / A F$ & lar \\
\hline $\mathrm{G} 18$ &.$/ 9 T^{\circ}$ & $9 / 1 \Delta$ & $N(\Delta)$ & .1 .1 & $1 / \cdot v$ & 199 & G46 & $V / e^{\circ} \Delta$ & FI/gr & ra/lv & . & ./aV & $|1|$ & G74 & $1 / r$ & IT/A. & IV/s. &.$/ r$ & $1 / \cdot \varphi^{c}$ & ז . & $\mathrm{G} 102$ & IN/N & Te/R & ITMT &.$/ 4{ }^{4}$ & .19. & 11. \\
\hline G19 & $1 / \cdot 0$ & IV/rT & $19 / \pi V$ & r & $1 / \cdot 1$ & $r .$. & G47 & VIAS & rNQD & $r 1 / . q$ & . & . $/ 9 T$ & $19 \mathrm{~V}$ & G75 & $\Delta /{ }^{\prime} \Delta$ & DT/S. & $\notin V / / \Delta$ & ז4א. & $V \cdot r$ & TrT & $\mathrm{G} 103$ & $\Delta / \cdot \Delta$ & INAD & rr/q. & $\cdot / N \Delta$ & $\cdot / A F$ & 191 \\
\hline $\mathrm{G} 20$ & 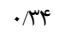 & $19 / \Delta \Lambda$ & $19 / \pi 4$ &.$/ .1$ & & Fes & G48 & $\vartheta / \odot$ & $\Delta \& / 9 V$ & rV/qT & $\cdot \mathrm{NA}$ & ./aV & 199 & G76 & $1 / 40$ & ISND & $\mid \omega / \pi$. &.$/ .{ }^{c}$ & $1 / .0$ & Trr & $\mathrm{G} 104$ & $r / \Lambda \Delta$ & INIA & سr/ &.$/ 1$ &.$/ 91$ & rI \\
\hline G21 & .Na & $r / \%$ & Tr/TA & r./. & $1 / 1$ & $r$. & G49 & $r / r$. & ז'וא & rT/QY & $\cdot M \Lambda$ & $1 / .0$ & TAY & G77 & T/ND & rNN. & $\Gamma \Delta /{ }^{\prime} \Phi \Delta$ &.$/ M V$ & 11.9 & Tas & G105 & $r / 9 \Delta$ & ISTS & $|r / \mu|$ &.$/$ & $\cdot / N V$ & TrV \\
\hline $\mathrm{G} 22$ &.$/ 99$ & $\mid r / . \cdot$ & $\mid r / \cdot \Delta$ &.$/ . r$ & 11.9 & $4 . q$ & G50 & $1 . / 1$. & $r V / r r$ & r &.$|9|$ & . Ar & $M \Lambda$ & G78 & $r / r$. & rY/NA & rNDA & $\cdot \pi V$ & $1 / \cdot{ }^{c}$ & TeV & G106 & .199 & $r \varphi / . \varphi$ & I ו &.$/ 0$ & I/T & זוץ \\
\hline $\mathrm{G} 23$ & $1 / r$ & 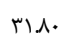 & $r \cdot / \Delta$. & .1 .9 & / & r ז & G51 & $9 /{ }^{2}$. & rGNT & TV/Tr & $\cdot / \Delta r$ & S & 190 & G79 & $r / r$. & $r \cdot / 9)$ & $T V / N$ & $\cdot / \Delta$ & $v \cdot r$ & Tr & G107 & $1 / . \cdot$ & TY.TY & rE/Tr &.$/ 4$ & $1 / 1$ & il. \\
\hline G24 & $9 / 90$ & $r \cdot \mu \Delta$ & $r N /$. & 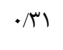 &.$/ 9$. & $1 \Lambda$. & G52 & $8 / \Delta \Delta$ & rq/4 & rT/NG & . &.$/ 99$ & 191 & G80 & $r / v$. & $\Delta T M$ & $\Delta * / .1$ & . & $1 / .9$ & $r \cdot 1$ & G108 & $1 / \pi \Delta$ & rNYA & TV/AT &.$/ . \Delta$ & $1 / 1$. & r \\
\hline $\mathrm{G} 25$ & $r / \Delta$. & $19 / A r$ & זrופו &.$M 1$ &.$/ 90$ & TFA & G53 & $N$. & $r \mu / \varphi^{c}$ & 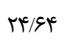 &.$N^{\infty} \Delta$ & . $/ A \Delta$ & $|D|$ & G81 & $r / N \Delta$ & $\Delta \Delta / / q a$ & $\Delta I / V^{C}$ & r & $V \cdot v$ & זצA & G109 & we. & 㗄 & Tr/FV &.$/ \cdot v$ & M. & rVr \\
\hline $\mathrm{G} 26$ & $1 / 4$ & WN. & $18 / \Lambda$ &.$/ .4$ & 11.9 & rVq & G54 & $\Delta / .$. & שתואז & INTH &.$/ \Lambda$ &.$/ 9$. & r.F & G82 & מזמ & $19 \mathrm{Na}$ & $10 / 4 T^{e}$ & 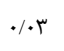 & 11.9 & זᄉ. & G110 &.$N$ & & $T r / 4 F$ &.$/ . r$ & $1 / 1$ & rwa \\
\hline G27 & $1 / \wedge \Delta$ & $19 / 44$ & $I V / \Delta q$ & $.1 . \Delta$ & $1 / * \varphi^{e}$ & זrT & G55 & $\Delta / T \Delta$ & rq/ז^ג & $r e / r r$ & | & $1 /$. & MM & G83 & IND & rNa. & $\Gamma V / \wedge \Delta$ & •ハ. & M. & req & G111 & $F / T \Delta$ & $r \mu / N$. & $r q / F \Delta$ &.$/ \pi \Lambda$ & $1 / .4$ & rFA \\
\hline G28 & $r / \mu$. & $\mid r / \Delta V$ & $1 . / T V$ & .1 .4 &.$/ 99$ & rav & G56 & $\Delta / 9 \Delta$ & $\mathrm{rV} / \mathrm{N}$ & TI/QT & זיא. &.$/ 91$ & 195 & G84 & $1 / \cdot$. & $r \Delta / / T V$ & $r \varphi / \mu v$ & $\% \vee v$ & V/r & एव, & G112 & r/q. & $r 91 . \mu$ & או/ & $\cdot \pi$ & $1 / \cdot 1$ & rqه \\
\hline
\end{tabular}


جدول ع- همبستخى بين عملكرد دانه و شاخصهاى تحمل به تنش در نمونهاى مورد مطالعه Table 4. Correlation between different tolerance indices and mean yield of samples used in this study

\begin{tabular}{|c|c|c|c|c|c|c|}
\hline TGSI & SSI & STI & TOL & Yn & Ys & \\
\hline & & & & & 1 & Ys \\
\hline & & & & 1 & $\cdot / \kappa \Delta^{* *}$ & Yn \\
\hline & & & 1 & $\cdot / N \cdot^{* * *}$ & $\cdot|r|^{* *}$ & TOL \\
\hline & & 1 & $\cdot / 4 f^{* * *}$ &.$|a|^{* * *}$ &.$/ 91^{* *}$ & STI \\
\hline & 1 & $-\cdot / r v^{* *}$ & $\cdot / \kappa^{* * *}$ & $\cdot|r|^{* * *}$ & $-\cdot \mid \Delta \Lambda^{* *}$ & SSI \\
\hline 1 & $\cdot \mid \Delta F^{* *}$ & $-\cdot \mid q \mu^{* * *}$ & $-\cdot / / V^{*}$ & $-\cdot / r v^{* * *}$ & $-\cdot|\Lambda|^{* *}$ & TGSI \\
\hline
\end{tabular}

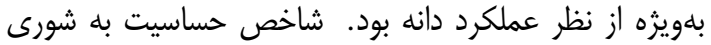

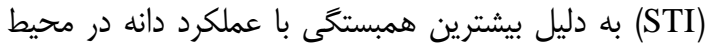

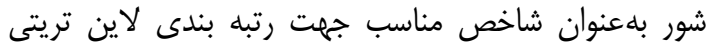

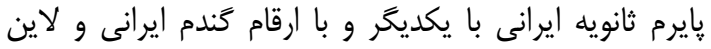

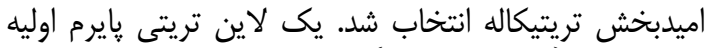
غير ايرانى

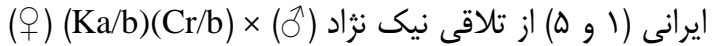

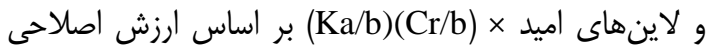

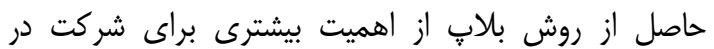

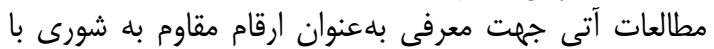

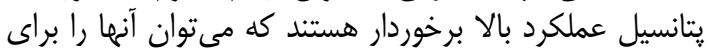
برنامههاى اصلاح رقم يِيشنهاد نمود.

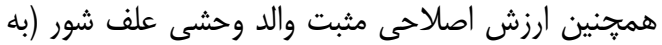

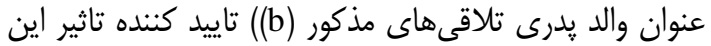

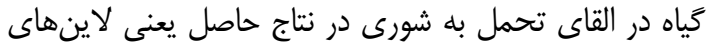

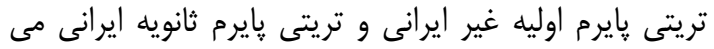

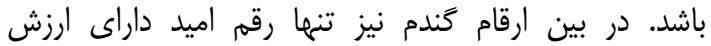
اصلاحى مثبت بود. اين در حالى است كه دو دو رقين رقم تترايلونئيد

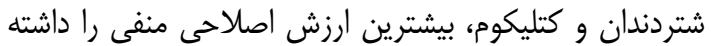

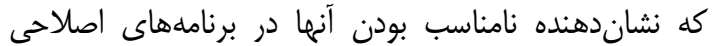

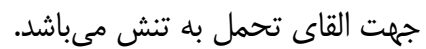

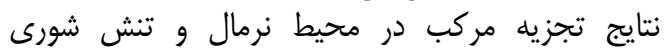
حاكى از وجود تنوع زيادى بين لاينهاى (EC=12 dS/m)

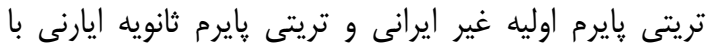

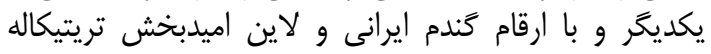

جدول ه- ارزش اصلاحى ييشبينى شده براى لاينهاى تريتى پايرم ثانويه ايرانى و والدين آنها Table 5. Predicated breeding value for ISTLs and Their parents

\begin{tabular}{|c|c|c|c|c|c|c|c|}
\hline ارزش اصلاحى & زنوتي؟ & ارزش اصلاحى & ز زنوتيت & اززش اصلاحى & زرنوتي" & اززش اصلاحى & زرنوتيب \\
\hline$\cdot 1+\cdot \mathrm{V}$ & $\mathrm{NKC} 12$ &.$- / 1$ & CM4 & $\cdot 1 \cdot 0$ & RA14 & $.1 \cdot 1$ & $\mathrm{~b}$ \\
\hline$\cdot 1 \cdot 1$ & $\mathrm{NKC} 13$ &.$- \cdot 1 \cdot 9$ & CM5 & $\cdot / \cdot r$ & RA15 &.$- / \cdot .9$ & $\mathrm{Ka}$ \\
\hline$-\cdot 1 \cdot V$ & SK1 & . & $\mathrm{OKC} 1$ &.- .1 .9 & RA16 &.$/ \cdots 1$ & $\mathrm{Cr}$ \\
\hline.$- / 11$ & SK2 & عس/. & OKC2 & $-\cdot 1 \cdot \Delta$ & RA17 & $\cdot 1 \cdot r$ & $\mathrm{Az}$ \\
\hline$-* / 11$ & SK3 & $\cdot / \mu \cdot$ & OKC3 & -.1 .9 & RA18 & $-\cdot / \cdot \varphi^{c}$ & $\mathrm{Ka} / \mathrm{b}$ \\
\hline$-+/ 1$ & SK4 & . Mt & OKC4 & $.1 \cdot 1$ & RA19 &.$-+1 \cdot 1$ & $\mathrm{Cr} / \mathrm{b}$ \\
\hline$-* / 1$ & SK5 & $\cdot / 4 V$ & OKC5 & $\cdot 1 \cdot 1$ & RA20 & .1 .4 & $\mathrm{Az} / \mathrm{b}$ \\
\hline \multirow[t]{22}{*}{.$- .1 .9$} & SK6 & צמא. & OKC6 & $.1+.1$ & RA21 &.$- \cdot 1 \cdot 1$ & $\mathrm{Ma} / \mathrm{b}$ \\
\hline & &.$/ 4 t$ & OKC7 & سו & FM1 & $\cdot / F \Delta$ & اميد \\
\hline & &.$/ 4 \Delta$ & OKC 8 & سו/.- & FM2 &.- .1 .9 & نيك نزاد \\
\hline & & צ צ/. & OKC9 & $-\cdot / 1 \pi$ & FM3 &.$- / 1 T$ & شتردندان \\
\hline & & • & OKC 10 & س & FM4 & $-. \cdot \cdot+r$ & روشن \\
\hline & & זس/. & OKC11 &.$- / 14$ & FM5 & $-\cdot \cdot \cdot 1$ & فلات \\
\hline & & שe & OKC 12 &.$- / 1 T$ & FM6 & $-\cdot / 11$ & كتليكوم \\
\hline & & $\cdot / M F$ & OKC13 & $-\cdot / \cdot v$ & FM7 & $\cdot / r \Lambda$ & $(\mathrm{Ka} / \mathrm{b})(\mathrm{Cr} / \mathrm{b})$ \\
\hline & & $\cdot / r \Lambda$ & OKC14 & $-\cdot / 1$ & FM8 &.$- \cdot 111$ & $(\mathrm{Ma} / \mathrm{b})(\mathrm{cr} / \mathrm{b})$ \\
\hline & & •/ & OKC 15 &.$- / 1 r$ & FM9 & $-\cdot \cdot \cdot r$ & RA1 \\
\hline & & $\cdot / \mu \cdot$ & OKC16 &.$- / 11$ & FM10 &.$- \cdot 1 \cdot \Delta$ & RA2 \\
\hline & & זr/. & $\mathrm{NKC1}$ & $-\cdot / 1 r$ & FM11 & $-\cdot / \cdot V$ & RA3 \\
\hline & & $\cdot 1 \cdot \varphi^{c}$ & $\mathrm{NKC2}$ &.$- / 1 T$ & FM12 & $-\cdot \cdot \cdot \cdot V$ & RA4 \\
\hline & & $\cdot 1 \cdot 1$ & NKC3 & س & FM13 & -.1 .9 & RA5 \\
\hline & & .1 .0 & NKC4 & سו/.- & FM14 &.$- \cdot \cdot r$ & RA6 \\
\hline & & $\cdot / T V$ & NKC5 & سו/.- & FM15 & $-\cdot \cdot \cdot 1$ & RA7 \\
\hline & & $\cdot / \cdots V$ & NKC6 & سו & FM16 & $-\cdot / \cdot r$ & RA8 \\
\hline & & $+\mid+1$ & NKC7 & سו/•- & FM17 & $-\cdot / \cdot \cdot r$ & RA9 \\
\hline & & $\cdot 1 \cdot \cdot V$ & NKC8 & سו & FM18 & $\cdot / \cdot \mu$ & RA10 \\
\hline & & $\cdot / \cdot r$ & NKC9 & $-\cdot / \cdot V$ & CM1 & .1 .9 & RA11 \\
\hline & & r.|. & NKC10 & $-\cdot 1 \cdot 1$ & $\mathrm{CM} 2$ & $-\cdot / \cdot \varphi$ & RA12 \\
\hline & & 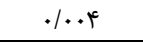 & $\mathrm{NKC} 11$ & -.1 .9 & $\mathrm{CM} 3$ & $-\cdot 1 \cdot 0$ & RA13 \\
\hline
\end{tabular}



-cross performance using RFLPs and information from related hybrids. Crop Science, 34: 20-25.

2. Falahatipour, S., H. Shahsavand Hassani, A. Baghizadeh and G. Karimzadeh. 2009. Characterization of secondary Tritipyrum introgressed genotypes by genomic DNA insitu hybridization technique. Journal of Science and Technology of Agriculture and Natural Resources, 13:65-74 (In Persian).

3. Falconer, D.S. and T.F.C. Mackay. 1996. The Introduction to quantitative genetics. $4^{\text {th }}$ edition. Longman group ltd, $144 \mathrm{pp}$.

4. Farshadfar, E., N. Mahmodi and A. Yaghotipoor. 2011. AMMI stability value and simultaneous estimation of yield and yield stability in bread wheat (Triticum aestivum L.). Australian Journal of Crop Science, 5: 1873-1844.

5. Fernandez, G.C.J. 1992. Effective selection criteria for assessing plant stress tolerance. In: Adaptation of food crop temperature and water stress. Proceeding of $4^{\text {th }}$ international symposium. Ed. Kus, E.G. Asian Vegetable Research and Department Center, Shanhua, Taiwan, 257-270pp.

6. Fernandez, R.J. and J.F. Reynolds. 2000. Potential growth and drought tolerance of eight deseat grasses. Journal Ecologia, 123: 90-98.

7. Fischer, R.A. and R. Maurer. 1978. Drought resistance in spring wheat cultivar. I. Grain yield responses. Australian Journal Agriculture Research, 29: 897-912.

8. Jahromi Razeghi, F., H. Shahsavand Hasani and A.H. Rezaei. 2011. Effect of salinity on yield and yield components of the primary lines of tritipyrum compared to wheat and triticale. Electronic Journal of crop production, 4: 1-16.

9. Kapoor, R., H. Evelin, P. Mathur and B. Giri. 2013. Approaches for abiotic stress tolerance in crop plants for sustainable agriculture. In: Tuteja N and Gill SS (eds) Plant acclimation to environmental stress. Springer, 359-401.

10. Khalifeie, N. and G. Mohammadi-Nejad. 2012. Evaluation of salt tolerance of new tritipyrum lines, triticale and Iranian wheat lines. Advances in Natural and Applied Sciences, 6: 206-212.

11. King, I.P., C.N. Law, K.A. Cant, S.E. Orford, S.M. Reader and T.E. Miller. 1997. Tritipyrum, a potential new salt-tolerant cereal. Plant Breeding, 116: 127-132.

12. Lauchli, A. and G. Grattan. 2007. Advances in molecular breeding toward drought and salt tolerance crops. Chapter1: Plant Growth and development under salinity stress. Springer, 1-32 pp.

13. Munns, R., R.A. James and A. Läuchli. 2006. Approaches to increasing the salt tolerance of wheat and other cereals. Journal of Experimental Botany, 57: 1025-1043.

14. Piepho, H.P., J. Mohring and A. Melchinger. 2008. BLUP for phenotypic selection in plant breeding and variety testing. Euphytica, 161: 209-228.

15. Poehlman, J.M. 2013. Breeding field crop. Scince, 724 pp.

16. Pourfereidouni, Z., H. Shahsavand Hassani and A. Baghizadeh. 2012. Study of Morphological Traits of Secondary Tritipyrum genotypes. American-Eurasian Journal Agricalture. and Enviroment. Science, 12: 259-261.

17. Sardouir-Nasab, S., G. Mohammadi-Nejad and B. Nakhoda. 2014. Field Screening of Salinity Tolerance in Iranian Bread Wheat Lines. Crop Science, 54: 1489-1496.

18. Shahsavand Hassani, H., P.D.S. Caligari, S.M. Reader, I.P. King and T.E. Miller. 2000. Can tritipyrum, a new salt tolerant potential amphiploid, be a successful cereal like triticale? Journal of Agricultural Science and Technology, 2: 177-195.

19. Shahsavand Hassani, H., Z. Roudbari and G. Mohammadi-Nejad. 2015. Evaluation of tolerance indices of tritipyrum lines comparison with wheat. The $2^{\text {th }}$ National Congress of Environmental Stresses in Plants. Kerman, Iran, 326-340 pp (In Persian).

20. Shahsevand Hassani, H., P.D.S. Caligari and T.E. Miller. 2006. Agronomical and adaptation characters of tritipyrum lines in comparison with triticale and Iranian wheat. Journal Plant Science, 5: 553-558.

21. Shannon, M.C. 1997. Adaptation of plant to salinity. Advances in Agronomy, 60: 87-120.

22. Thomason, W.E. and S.B. Phillips. 2006. Methods to evaluate wheat cultivar testing environments and improve cultivar selection protocols. Field Crop Research, 99: 1-9.

23. Villumsen, T.M. and L. Janss. 2009. Bayesian genomic selection: the effect of haplotype length and priors. BMC Proc 3 Suppl, 1-11 pp. 


\title{
The Estimation of Breeding Value of Iranian Secondary Tritipyrum based on Stress Tolerance Indices by Best Linear Unbiased Prediction
}

\section{Zahra Roudbari ${ }^{1}$, Hossein Shahsavand Hassani ${ }^{2}$ and Ghasem Mohammadi-Nejad ${ }^{3}$}

1 and 3- PhD Student and Associate Professor, College of Agriculture, Shahid Bahonar University of Kerman

2- Associate Professor, College of Agriculture, University of Shiraz, Shiraz, (Corresponding Authore: Shahsavand@shirazu.ac.ir)

\author{
Receive: October 23, $2016 \quad$ Accepted: February 19, 2017
}

\begin{abstract}
Although the non-Iranian primary tritipyrum lines (NIPTLs) have been produce and have shown potential to be a new slat tolerant cereal, however, have a few undesirable traits such as brittle rachis, keep in tiller production and late maturity. In order to remove these traits, the crossing of NIPTLs with Iranian bread wheat cultivars led to new recombinant Iranian secondary tritipyrum lines. In this study the 13 non-Iranian primary tritipyrum lines (NIPTLs: $2 \mathrm{n}=6 \mathrm{x}=42 ; \mathrm{AABBE}^{\mathrm{b}} \mathrm{E}^{\mathrm{b}}$ ) and Iranian secondary tritipyrum lines (ISTL: $\mathrm{F}_{6}-\mathrm{F}_{7} ; 2 \mathrm{n}=6 \mathrm{x}=42$, $\left.\operatorname{AABBD}_{(1-6)} \mathrm{E}_{(1-6) ")}\right), 6$ Iranian bread wheat cultivars and 1 promising triticale line were evaluated at two research centers using an alpha lattice design with two replications under normal $\left(1 \mathrm{dS} \mathrm{m}^{-1}\right)$ and salinity stress $\left(12 \mathrm{dS} \mathrm{m}^{-1}\right)$ conditions during growing seasons of 20142016 at Kerman province of Iran. The results indicated grain yield was strongly affected by salinity with reduction ratio of $11.06,9.37,4.30,4.98,7.98,12.58$, in Iranian chromosomally recombinant secondary individuals tritipyrum plants obtained from crosses such as (Cathlicum $\times \mathrm{Ma} / \mathrm{b}),($ Falat $\times \mathrm{Ma} / \mathrm{b}),\{$ Omid $\times(\mathrm{Ka} / \mathrm{b})(\mathrm{Cr} / \mathrm{b})\},\{$ Niknejad $\times(\mathrm{Ka} / \mathrm{b})(\mathrm{Cr} / \mathrm{b})\},($ Shotordandan $\times$ $\mathrm{ka} / \mathrm{b})$, (Roushan $\times \mathrm{Az} / \mathrm{b})$, respectively, in comparison with Non Iranian Primay Ttritipyrum Lines (NIPRLs) (3.48), Iranian bread wheat cultivars (19.90) and promising triticale line (15.87) The evaluation of stress tolerance indices showed that STI could effectively be used for screening of salinity tolerant genotypes because it had the highest correlation coefficients with grain yield. The $(\mathrm{Cr} / \mathrm{b}) \times(\mathrm{Ka} / \mathrm{b})$ line of NIPTLs and the lines obtained from Niknejad $\times$ $(\mathrm{Ka} / \mathrm{b})(\mathrm{Cr} / \mathrm{b})$ and Omid $\times(\mathrm{Ka} / \mathrm{b})(\mathrm{Cr} / \mathrm{b})$ crosses showed the highest average breeding value which will be suitable for breeding programs of ISTLs lines with high yield potential in saline soils and brachish waters.
\end{abstract}

Keywords: Best linear unbiased prediction (BLUP), Iranian secondary tritipyrum lines (ISTL), Non-Iranian primary tritipyrum lines (NIPTL), Salt stress, Stress tolerance indices 